- The quality of processing is critical to the effective use of radiographs.

- A large proportion of this group of GDPs were producing radiographs which had been processed in weak solutions.

- The point at which solutions require changing is difficult to predict using either time or numbers of radiographs processed.

- Use of a simple monitoring device enhances quality assurance in dental radiography.

\title{
Assessing the quality of radiographic processing in general dental practice
}

\author{
P. H. Thornley, ${ }^{1}$ D. A. Stewardson, ${ }^{2}$ P. G. J. Rout ${ }^{3}$ and F. J. T. Burke ${ }^{4}$
}

\begin{abstract}
Objectives To determine if a commercial device (Vischeck) for monitoring film processing quality was a practical option in general dental practice, and to assess processing quality among a group of GDPs in the West Midlands with this device.

Design Clinical evaluation.

Setting General dental practice, UK, 2004.

Method Ten GDP volunteers from a practice based research group processed Vischeck strips (a) when chemicals were changed, (b) one week later, and (c) immediately before the next change of chemicals. These were compared with strips processed under ideal conditions. Additionally, a series of duplicate radiographs were produced and processed together with Vischeck strips in progressively more dilute developer solutions to compare the change in radiograph quality assessed clinically with that derived from the Vischeck.

Results The Vischeck strips suggested that at the time chosen for change of processing chemicals, eight dentists had been processing films well beyond the point indicated for replacement. Solutions were changed after a wide range of time periods and number of films processed. The calibration of the Vischeck strip correlated closely to a clinical assessment of acceptable film quality.

Conclusions Vischeck strips are a useful aid to monitoring processing quality in automatic developers in general dental practice. Most of this group of GDPs were using chemicals beyond the point at which diagnostic yield would be affected.
\end{abstract}

\section{INTRODUCTION}

The United Kingdom Department of Health has stated that strict attention should be paid to correct and consistent film processing in order to produce good quality radiographs and reduce the necessity for examinations to be repeated. ${ }^{1}$ While any exposure to radiographs is believed to carry a risk of inducing cancer, dental radiography involves low doses and associated risks, but is a

\footnotetext{
${ }^{1}$ General Dental Practitioner, Sutton Coldfield, Birmingham: ${ }^{2 *}$ Clinical Lecturer Conservative Dentistry, Birmingham, St Chad's Queensway Birmingham B4 6NN; ${ }^{3}$ Consultant in Oral Radiology, Birmingham Dental Hospital, Birmingham; ${ }^{4}$ Professor of Primary Dental Care, University of Birmingham, School of Dentistry, Birmingham / South Birmingham Primary Care Trust

*Correspondence to: Dominic Stewardson

Email:d.a.stewardson@bham.ac.uk
}

\section{Refereed paper}

Accepted 15 June 2005

doi: $10.1038 /$ sj.bdj.4813527

๑ British Dental Journal 2006; 200: 515-519 high volume investigation. Nevertheless, the effective dose from dental radiography, for example, is reported to range from 1 to $8.3 \mu \mathrm{Sv}$ for an intra-oral radiograph and from 3.85 to $30 \mu \mathrm{Sv}$ for panoramic films. The estimated risks of developing a fatal malignancy with these doses range from 0.02 to 0.06 per million and from 0.2 to 1.9 per million examinations respectively. ${ }^{2}$

In a study looking at the quality of panoramic radiographs in a sample of general practices in the UK, 33\% were found to be of unacceptable diagnostic quality, and $40 \%$ had processing faults of density or contrast. ${ }^{3}$ A study by the authors ${ }^{4}$ examining bitewing radiographs taken in general dental practice showed a wide variation in the processing quality of radiographs. Possible reasons for poor quality include expired chemicals and films, processing temperature variations due to varying ambient temperatures with manual processing or thermostat faults on automatic processors, light contamination and transport mechanism faults.

The Ionising Radiation (Medical Exposure) Regulations 2000 (IRMER $)^{5}$ require users of ionising radiation to pay special attention to quality assurance (QA). With regard to radiographic processing, a QA programme should be in place to monitor radiographic quality. As part of this mechanism, routine checks should be made to ensure that darkrooms and desktop automatic processing units remain light tight, to prevent film fogging. In addition, the overall performance of processing should be monitored. The National Radiological Protection Board (NRPB) guidelines ${ }^{1}$ suggest that a simple way of achieving this is with the use of a testobject such as a step-wedge. In hospital radiology departments, QA programmes for radiographic processing use relatively expensive sensitometric and densitometric techniques, but Rushton and Horner showed that a simple homemade step-wedge device can be effective in general dental practice. ${ }^{6}$ They also showed that introducing processing QA procedures helped reduce unacceptable films taken in general dental practice from 49\% to 39\%, although these figures were still well above the NRPB target that only $10 \%$ should be unacceptable. ${ }^{7}$

Other ways of reducing processing errors could be to move away from chemical processing altogether and introduce digital radiography. The cost of these techniques suggests that it will be some time before they are widely used in the UK, and figures are not available to show the current level of UK usage. However, a recent study in Sweden showed that 13\% of 14,624 radiographs submitted to a dental insurance office were taken with a digital technique. ${ }^{8}$ Interestingly, significantly more errors were found in 


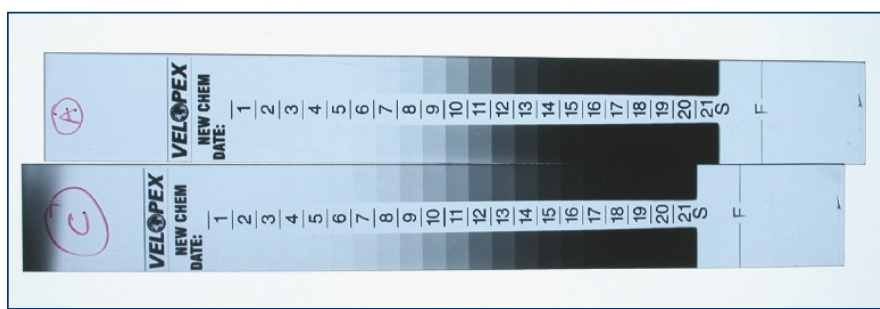

Fig. 1 Vischeck strip processed in new chemicals $(A)$ is used as a reference strip and compared with strips processed subsequently. Strip C (test strip) is aligned with strip $A$ by bringing a bar of similar density alongside bar number 12 on the reference strip. The need to change processing solutions is indicated when there is a difference of greater than two steps between the test and the reference strip. Fogging can be seen at the left hand edge of strip $C$.

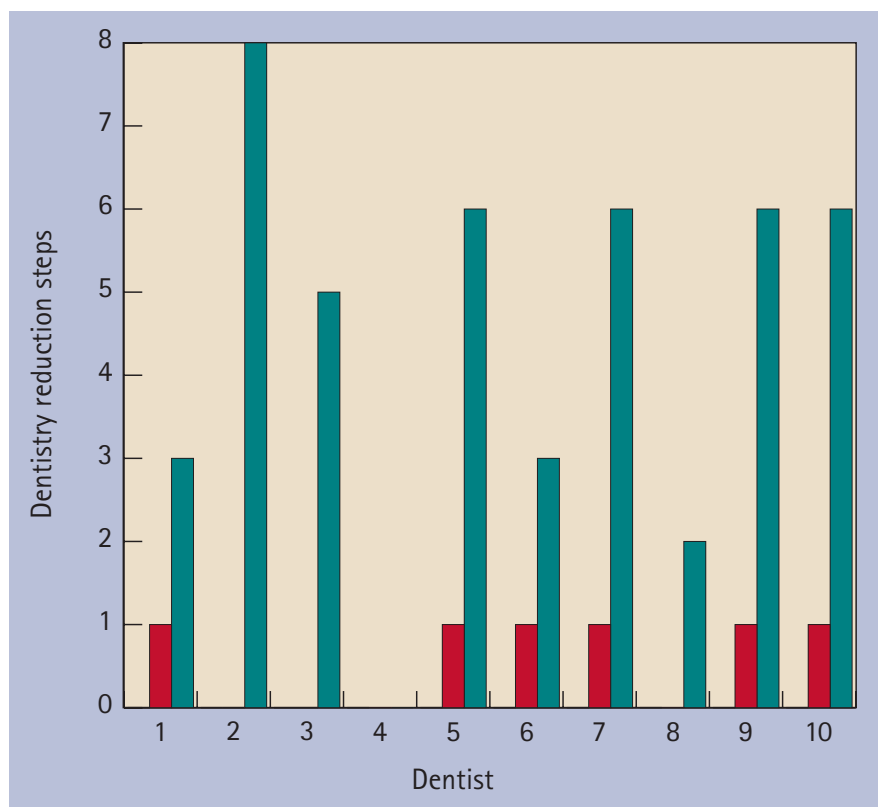

Fig. 2 Reduction in density of Vischeck strips at baseline (initial), after one week and immediately before the next change of developing solutions (final).

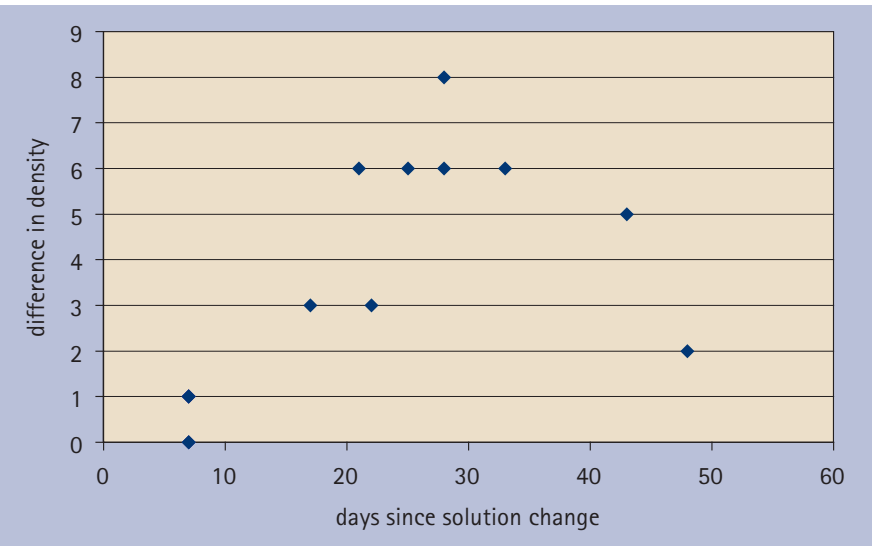

Fig. 3 Plot of difference in density of Vischeck strips against time since change of processing solutions.

the intra-oral digital radiographs compared to the radiographs taken with x-ray film. Many of these were related to the positioning of the digital receptors.

A disadvantage of the step-wedge technique for monitoring processing quality is that it involves a radiographic exposure of a film beneath the wedge. This means that an additional variable, the exposure of the film is introduced into the process. The repeated task of exposing test films may also be a disincentive to carrying out the procedure in a busy dental practice.

Recently, a manufacturer of automatic film processors
(Medivance Instruments Ltd, Harlseden, London, UK) has developed a reference system called Vischeck to monitor the quality of radiographic processing within dental practice. This consists of a box containing pre-exposed film strips. One is used as a reference or control strip and is developed immediately after the automatic processor solutions have been changed. This produces an image containing a scale of 21 bars of different grey shades. This is then compared to subsequent test strips processed in the dental practice, ideally on a daily basis. The two strips are aligned and bar no 12 on the reference strip is aligned with a bar that has the same density on the test strip (Fig. 1). The ideal is that both bands number 12 should have the same density. If the bands of equal density are more than two numbers apart between the test strip and the control strip, then the chemicals are outside the recognised limits (according to the manufacturer) for processing of radiographic film. Provided the developer temperature is within correct limits, this indicates that the chemicals need to be changed. (In processing machines which have an automatic system for replenishment of chemicals, a discrepancy between reference and test strip may indicate failure of the replenishment mechanism, empty replenishment reservoirs or exhausted chemicals in the reservoirs). Such a device may provide a relatively inexpensive and easy means of monitoring processing quality and achieving quality assurance (current manufacturer's price is £38 per box of 24 strips). However, it has not been established that the level determined by the Vischeck as indicating that chemicals should be changed would agree with a clinical decision made by assessing radiograph image quality.

\section{OBJECTIVES}

The objectives of this study were to determine if the Vischeck strips were a useful and user-friendly adjunct to monitoring film processing quality in general dental practice, to assess the film processing quality of a group of general dental practices in the West Midlands by this method, and to compare the Vischeck with visual assessment of radiographs.

\section{METHOD}

\section{a. General dental practices}

Ten general dental practitioners (GDPs) from different dental practices within the West Midlands were recruited from a research study group associated with the University of Birmingham School of Dentistry (BRIDGE - Birmingham Research in General Dental Practice - www.gdp-research.org.uk). Each practitioner was provided with three Vischeck strips, a written protocol and a data collection sheet. Nine of the practitioners used an automatic Velopex Extra-X machine (Medivance Instruments Ltd, Harlseden, London, UK) and one used manual processing procedures. The practitioners were asked to develop one strip immediately after changing the solutions in their processing units. They were to develop the second strip exactly one week later and the third strip was to be developed immediately before the solutions were changed. The timing of the change of chemicals was determined by the dentist according to their own criteria. The dentists were asked to record the temperature of their developing solutions, asked the dates the strips were developed, the dates the solutions were changed and the temperature of their developing and fixing solutions each time the strips were developed. The numbers and types of diagnostic radiographic films taken between each stage were also recorded. The dentists were also asked to record the brand and type of the solutions used in their developing and fixing tanks, and the concentration.

This protocol would permit the quality of processing to be evaluated at an optimal level, and at its lowest level ie immediately after solution change and immediately before the next change when solutions are less effective. Assessing quality at a point one week after the solutions have been changed should give an indica- 


\begin{tabular}{|c|c|c|c|c|c|c|c|c|c|c|}
\hline & & A & & & B & & & C & & \\
\hline & & INITIAL & & & NE WE & & & FINAL & & \\
\hline DENTIST & $\begin{array}{c}\text { Density } \\
\text { difference } \\
\text { (steps) }\end{array}$ & fog & $\begin{array}{c}\text { Films } \\
\text { processed }\end{array}$ & $\begin{array}{c}\text { Density } \\
\text { difference }\end{array}$ & fog & $\begin{array}{c}\text { Films } \\
\text { processed }\end{array}$ & $\begin{array}{c}\text { Density } \\
\text { difference }\end{array}$ & fog & $\begin{array}{c}\text { Films } \\
\text { processed }\end{array}$ & $\begin{array}{c}\text { Time } \\
\text { (days) }\end{array}$ \\
\hline Ref BDH & 0 & 0.18 & 0 & 0 & 0.18 & 0 & 0 & 0.17 & 0 & 0 \\
\hline 1 & 0 & 0.18 & 0 & -1 & 0.17 & 45 & -3 & 0.18 & 120 & 22 \\
\hline 2 & 0 & 0.19 & 0 & 0 & 0.18 & 27 & -8 & 0.17 & 42 & 28 \\
\hline 3 & 0 & 0.18 & 0 & 0 & 0.19 & 55 & -5 & 0.17 & 77 & 43 \\
\hline 4 & 0 & 1.9 & 0 & & 1.63 & & & 1.69 & & \\
\hline 5 & 0 & 0.52 & 0 & -1 & 0.17 & 57 & -6 & 0.24 & 183 & 28 \\
\hline 6 & 0 & 0.18 & 0 & -1 & 0.19 & 42 & -3 & 0.17 & 75 & 17 \\
\hline 7 & 0 & 0.28 & 0 & -1 & 0.19 & 57 & -6 & 0.18 & 355 & 33 \\
\hline 8 & 0 & 0.34 & 0 & 0 & 0.34 & 20 & -2 & 0.18 & 73 & 48 \\
\hline 9 & 0 & 0.18 & 0 & -1 & 0.17 & 35 & -6 & 0.17 & 170 & 25 \\
\hline 10 & 0 & 0.17 & 0 & -1 & 0.17 & 31 & -6 & 0.17 & 81 & 21 \\
\hline \multicolumn{11}{|l|}{ TOTALS } \\
\hline 0 steps & 10 & & & 4 & & & 1 & & & \\
\hline 1 step & 0 & & & 6 & & & 0 & & & \\
\hline 2 steps & 0 & & & 0 & & & 1 & & & \\
\hline 3 steps & 0 & & & 0 & & & 2 & & & \\
\hline$>3$ steps & 0 & & & 0 & & & 6 & & & \\
\hline
\end{tabular}

tion of the rate of decline in quality. The dentists labelled their strips A, B and C referring to those processed at baseline, after one week and immediately before the next change of processing solutions. They were returned with the data collection forms. A consultant oral radiologist then examined the strips in low ambient light on a small light box.

\section{b. Batch testing}

Three reference strips from different batches of Vischeck strips were also developed under ideal conditions using fresh solutions at Birmingham Dental Hospital, under the supervision of a Consultant Radiologist. The purpose of these strips was to provide an external reference strip against which the GDPs monitoring strips could be checked. It was also possible to ascertain if there was any initial difference between the three boxes of Vischecks supplied to the dentist.

The three reference strips developed in the dental hospital processor (Durr XR 24 Nova, Durr Dental, Wellingborough, Northants, UK) were examined first, using the method described above. The first strip was placed on the light box, and the other batch control strips placed next to it. All bands number 12 aligned, ie there was no observable difference in density between them. Therefore, comparing strips from the different dentists who had used strips from different batches was justified by this uniformity. Each set of the 10 GDPs' films was then examined.

The area of the Vischeck strip beyond the reference bars should be clear on the test and control strips. Darkening in this area, (fogging) indicates light contamination of the strip, and this will affect the accuracy of the strip as a whole. To test for potential areas of increased film fogging during film handling of the test strip, the base plus fog levels (background film fogging) were measured. This was performed with an X-rite 331 densitometer (X-Rite Ltd, Poynton, Cheshire, UK). A reading greater than 0.20 indicates increased levels of fogging (NB film density has no units). The films from dentist 4 (developed manually) all showed excessive fogging making them unsuitable for inclusion in this study.

The strips developed at the dental hospital were given the reference BDH, and those of the dentists were given references 1-10. All dentists used Medivance Extra-X Velopex machines, except dentist 4, who developed films manually in a dark room.

\section{c. Visual radiograph assessment vs vischeck assessment}

To compare radiographic quality with the Vischeck, a beam aiming device (Rinn Dentsply, Weybridge, Surrey, UK ) was located on a dried dentate mandible using a rigid silicone material (Virtual Bite Registration Silicone, Ivoclar-Vivadent, Enderby, Leicester, UK) to allow repeated periapical radiographs of the molar region to be taken with reproducible positioning of the $\mathrm{X}$ ray tube, film and target teeth relative to each other. By this method, a series of radiographs were obtained of the right and left side on Kodak F Speed film (Kodak UK, Hemel Hempstead, Herts, UK), using a Dental EZ HDX X-ray unit (DentalEZ dental products (GB), Hemel Hempstead, Herts, UK) at the same exposure recommended by the manufacturer of this X-ray machine. One pair of exposed films, and a Vischeck strip were processed in a Velopex Extra-X automatic processor containing fresh chemicals and operating at the recommended temperature. These would serve as ideal baseline references. The developer was then diluted with distilled water to produce solutions with strengths of $80 \%$, $40 \%, 20 \%, 10 \%$ and 5\% of the original. This would decrease the activity of the developer in a controlled fashion and simulate the deterioration that would occur in practice through use and passage of time. Pairs of films with a Vischeck strip processed in each of the weakened solutions at the recommended temperature were then viewed as before to identify at what point radiographic quality had noticeably deteriorated. The related Visicheck strips were then aligned and compared as described above.

Data were entered into a computer spreadsheet programme for analysis (Microsoft Excel, Microsoft Corp, USA).

\section{RESULTS}

Figure 2 shows the change in density in terms of steps on the Vischeck scale at the three evaluation points. Table 1 records the measured background fogging, the numbers of radiographs taken at one week and at the final evaluation, and the number of days between the baseline and final evaluation. There was no absolute correlation between the number of days between developer changes and the deterioration in test strip quality; dentist 10 went 21 days between solution change but had reduced density of six units, whereas dentist 1 went 22 days but 


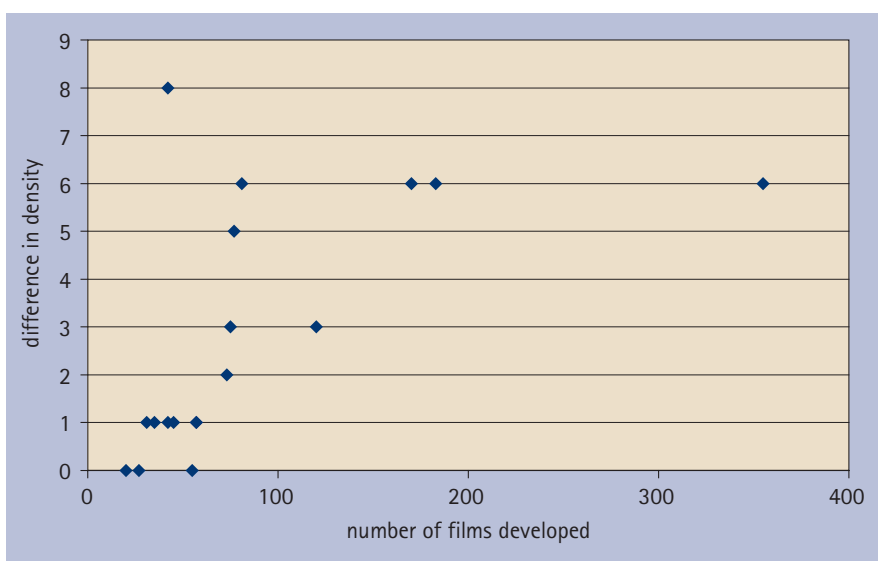

Fig. 4 Plot of difference in density of Vischeck strips against number of films processed.

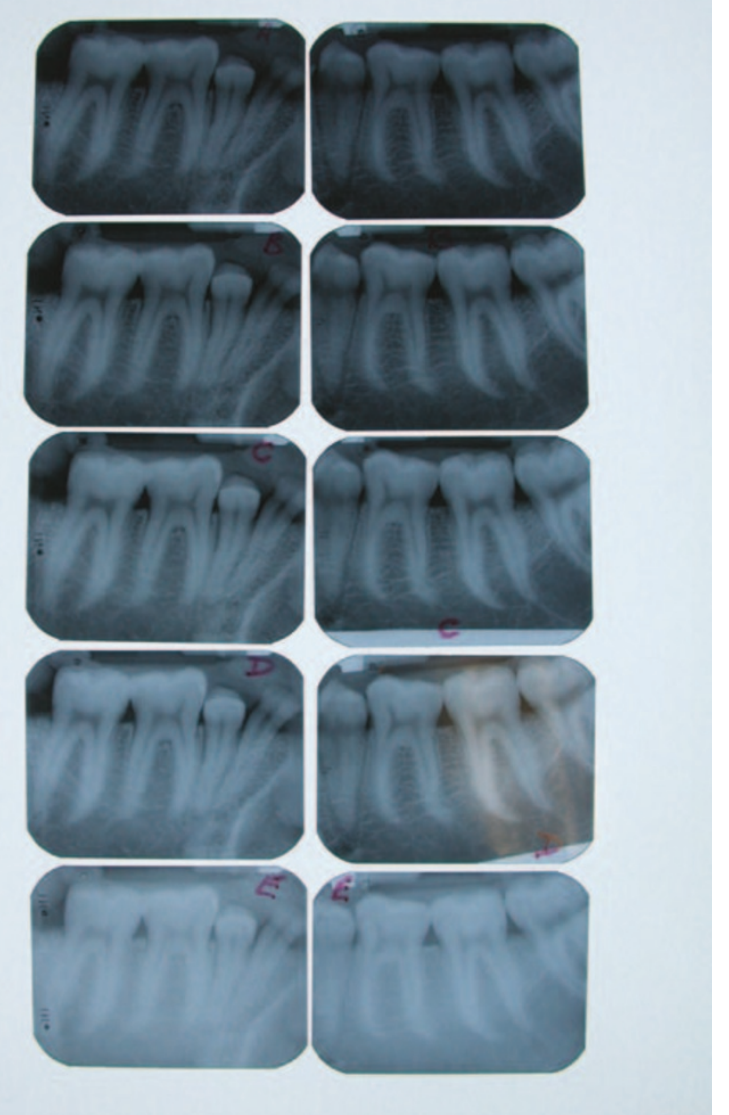

Fig. 5 Film pairs produced at dilutions of $100 \%, 80 \%, 40 \%, 20 \%$ and $10 \%$.

only had reduction of three units (Fig. 3). However, the longer the time between solution change, the greater the deterioration in check strip density. Similarly there was a general trend of reduced density as more films were developed, but again the relationship was not linear (Fig. 4). Of those dentists using automatic developers, all except one (dentist 9) used the same make and concentration of fixer and developer solution.

Two of the authors independently examined the films and Vischeck strips processed at differing dilutions. There was complete agreement regarding the point at which film quality deterioration was both noticeable and was affecting diagnostic yield. Figure 5 shows the film pairs processed in solutions of 100\% (A), 80\% (B), 40\% (C), 20\% (D) and 10\% (E). Film pair F (5\%) was of such a poor quality that formal assessment was not necessary. Films A, B and C were subjectively judged to be of sufficient quality for clinical diagnosis and beyond this stage solution change would be required (ie film pair D). Alignment of the corresponding test Vischeck strips with the baseline strip processed at full developer strength showed that by strip C processed at $40 \%$ dilution, only a one step difference had occurred, but by strip D (20\%), a three-step difference had occurred (Fig. 6).

\section{DISCUSSION}

Loss of radiographic density reduces the diagnostic yield of radiographs. ${ }^{9}$ Density is primarily determined by exposure factors. ${ }^{9}$ Underdevelopment, however, can also produce a radiograph with reduced density. It is therefore essential that deterioration of the processing solutions is identified to ensure that diagnostic radiographs are obtained. This study has shown the value of monitoring film processing.

It is acknowledged that the number of dentists in this study was small, however, it is felt that inferences can be drawn from the results because of the general constancy of the findings.

It can be seen from the results for the test reference strips developed in the dental hospital processing unit, that there was consistency between different batches of Vischeck strips (Table 1). Indeed, for all the strips developed in automatic developers by nine dentists, there was no difference in density immediately after solution change. Of those developed immediately after solution change (the films processed automatically in group A), three out of nine films had some evidence of fogging (Table 1). However, the fogging did not affect the step densities, being localised to one edge of the strip. The background fog reading was taken at the worst area on the film, and was higher than the recommended 0.20 units (read on the densitometer) for these three films.

Interestingly, the number of films with background fog reduced for group B (developed after one week) and was lowest for group C (developed immediately before solution change). This could indicate that there is a learning curve associated with handling the check strips. The test strip is sealed in a thick plastic envelope which requires opening with scissors within the limited confines of the daylight loader. It was thought that the first set of films may have been contaminated by light due to inexperience in opening the film packets. It is suggested that the design of the packets is changed to allow for easier opening. The excessive fogging seen in those films processed manually (dentist 4) is of concern, as it will have a serious effect on the diagnostic worth of the radiographs. It was likely that safelight conditions had been inappropriate.

Of those films developed after one week (group B), three of the nine strips had no difference in density compared to the reference strips. Six strips had slight deterioration, but these showed only one unit change and thus were still diagnostically acceptable, as recommended by the manufacturer. There was a considerable difference in the number of films developed by the different surgeries, ranging from a minimum of 20 films developed in the week, to a maximum of 57 films. However, there was no correlation between the number of films developed and the degree of reduction in density of the test strips, indicating the strength of the developer solution had not been significantly depleted.

For group C (those films developed immediately before solution change), only one dentist in nine produced a strip that was within the recommended two units of density change. That is to say, eight out of the nine dentists were using exhausted chemicals to develop their radiographs. Two dentists produced check strips underdeveloped by three grades, and six dentists produced check strips underdeveloped by four or more units. One dentist was eight grades underdeveloped, and it is likely that any x-ray films developed in this processor would have become pale, lacking contrast and density resulting in loss of diagnostic information. As a consequence, there may have been the temptation to increase the exposure time to compensate for the pale films. There was wide variation in the number of days between solu- 


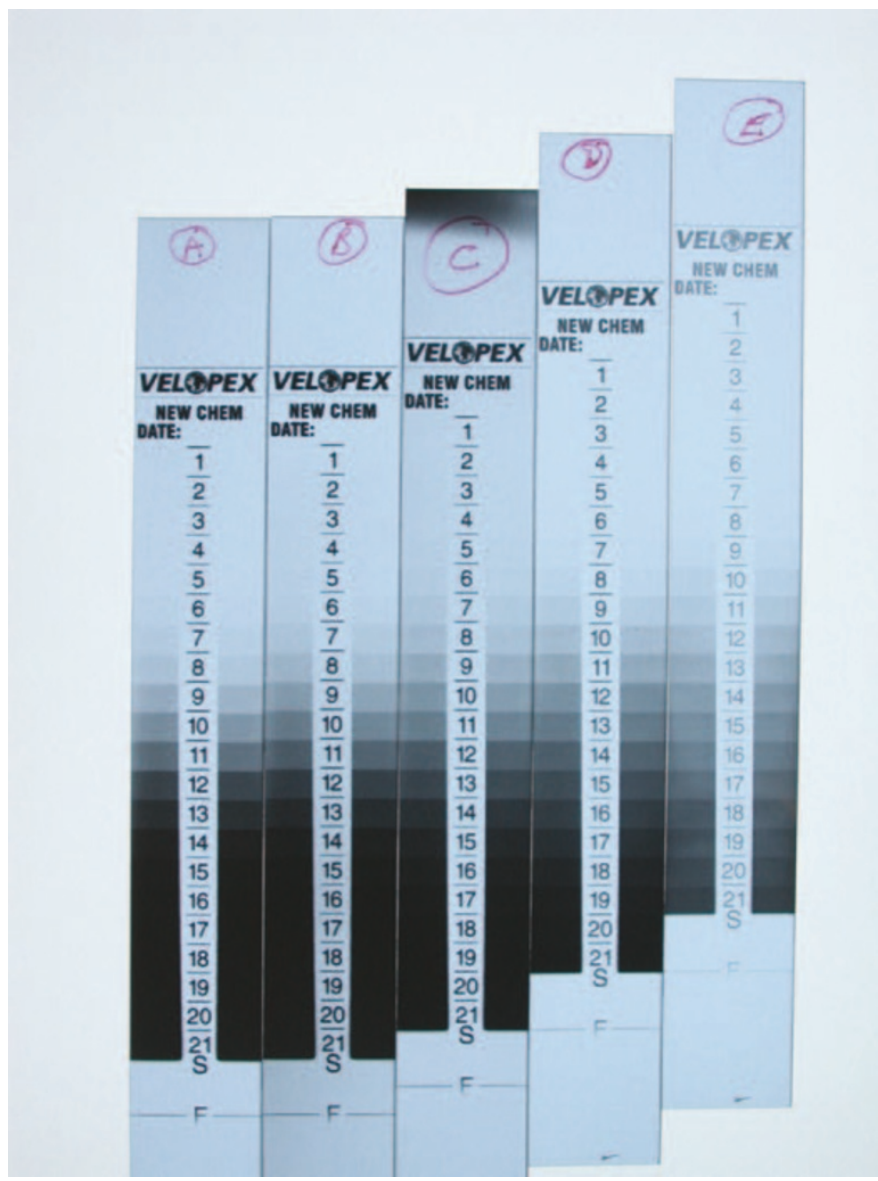

Fig. 6 Vischeck strips processed at dilutions of 100\%, 80\%, 40\%, 20\% and $10 \%$ aligned for comparison. A one step difference has occurred with strip C and a three-step difference with strip D.

tion change in the different surgeries. The shortest time was 17 days and the longest time was 48 days.

The results showed that there was a marked reduction in strip density between one and three weeks. However, the results also indicated that the actual cut-off time cannot be determined by either passage of time or number of films developed. For this reason, the use of Vischeck strips or a test wedge on a daily basis particularly between the second and third week of solution change is to be recommended.

By comparing both clinical radiographs and Vischeck strips processed with depleted developer, it may be seen that there is good agreement between the indication given by the Vischeck strips of when to change chemicals with that which could be determined by clinical criteria.

\section{CONCLUSIONS}

Within the confines of this small study, it may be concluded that the use of reference developing check strips appears to be a useful aid to monitoring processing quality in automatic developers in general dental practice. The strips are less time consuming to use than a home made step-wedge, are more accurate due to the greater number of step densities and remove the variable of the exposure setting of the $\mathrm{x}$-ray tube. In addition, the calibration of the strips relates well with diagnostic acceptability.

It is recommended that the design of the Vischeck strip packet is improved to enable easier opening in the automatic developer.

There was wide variation between the length of time and number of diagnostic films that GDPs process before solutions were changed. Only one dentist changed the chemicals in his developer before the solution became too exhausted to ensure less than two units change in density as measured by Vischeck strips and only three out of 10 dentists changed solutions at three units change in density. The majority of this group therefore are not changing their chemicals early enough and this will result in the production of radiographs with reduced diagnostic yield which may impact on the treatment of their patients. A manually processed film had severe background fogging and the darkroom should be investigated for light contamination.

The authors would like to thank the participating GDPs for their co-operation, and Medivance, the manufacturers of Vischeck, for providing the test strips.

1. Guidance notes for dental practitioners on the safe use of $x$-ray equipment. Didcot: Department of Health and NRPB, 2001

2. Pendlebury $M$, Horner $K$, Eaton $K$ (Eds). Selection criteria for dental radiography. $2^{\text {nd }}$ edn. p 16. UK: Faculty of General Dental Practitioners, 2004.

3. Rushton $\mathrm{V} E$, Horner $\mathrm{K}$, Worthington $\mathrm{H} \mathrm{V}$. The quality of panoramic radiographs in a sample of general practices. Br Dent J 1999; 186: 630-633.

4. Thornley P, Stewardson D, Rout J, Burke F J T. Rectangular collimation and radiographic efficacy in eight general dental practices in the West Midlands. Prim Dent Care 2004; 11: 81-86.

5. The lonising Radiation (Medical Exposure) Regulations 2000, SI 2000 No. 1059, HMSO, London.

6. Rushton V E, Horner K. A laboratory evaluation of four quality control devices for radiographic processing. J Dent 1994; 22: 213-222.

7. Rushton $V E$, Horner $K$. The impact of quality control on radiography in general dental practice. BrDent J 1995; 179: 254-261.

8. Hellen-Halme K, Johansson P M, Hakansson J, Petersson A. Image quality of digital and film radiographs in applications from the Dental Insurance Office in Sweden for treatment approval. Swed Dent J 2004; 28: 77-84.

9. Langland 0 , Langlais R, Preece J. Principles of dental imaging. $p 53.2^{\text {nd }}$ edn. Baltimore: Lippincott Williams \& Wilkins, 2002.

\title{
BDJ Correction
}

\section{Vol 200 No. 7, April 8 2006, cover}

\author{
In Volume 200 issue 7 of the BDJ the third cover line given was Two implants for all edentulous \\ mandibles. This article did not appear in issue 7 and was published in issue 8 on April 22 2006, p 469.
}

